\title{
Study of inertia effect on thermohydrodynamic characteristics of Rayleigh step bearings by CFD method
}

\author{
M. Vakilian, S.A. Gandjalikhan Nassab ${ }^{\mathrm{a}}$ And Z. Kheirandish \\ Mechanical Engineering Department Shahid Bahonar University Kerman, Iran
}

Received 30 January 2013, Accepted 11 June 2013

\begin{abstract}
This paper presents a numerical study about lubricant inertia effect on thermohydrodynamic (THD) characteristics of Rayleigh step bearings running under steady, incompressible and laminar condition. To reach this goal, the set of governing equations is solved numerically with and without considering the inertia terms. The discretized forms of the momentum and energy equations are obtained by the finite volume method and solved using the Computational Fluid Dynamic (CFD) technique. These equations are solved simultaneously because the dependency of lubricant viscosity with temperature. The hydrodynamic and thermal behaviors of the slider step bearings are demonstrated by presenting several figures including the lubricant pressure and temperature distributions with and without considering the fluid inertia effect. Numerical results show that inertia term has considerable effect on THD characteristics of step bearings, especially when they run with high velocity of runner surface.
\end{abstract}

Key words: Rayleigh step bearing / Inertia / CFD / THD

\section{Introduction}

Hydrodynamic step bearings are widely used in industries to support axial loads efficiently. Many type of step bearings were theoretically studied in the 1950 s by researchers in order to predict and improve their performances.

In 1962, Dowson [1] introduced the generalized Reynolds equation, which allows for cross-film temperature variations. This equation was solved with realistic THD boundary conditions by Ezzat and Rohde [2] using the finite difference method. In 1986, Boncompain et al. [3] improved the numerical model by considering reverse flow, fluid-film rupture and elastic deformations (Thermoelastohydrodynamic solution). Auloge et al. [4] studied the optimum design of Rayleigh step bearing and determined the relationships between step location and height along with non-Newtonian lubricants. The same method was used by Fillon and Khonsari [5] in tracing design charts for tilting-pad journal bearings. In 2002, Arghir et al. [6] used a Reynolds-based numerical method applicable to discontinuous geometrical domains, which included a concentrated inertia effect model. The complete energy and Navier- Stokes equations were solved using the finite volume method and the SIMPLEC algorithm. In that work, the discontinuity in film thickness,

\footnotetext{
${ }^{a}$ Corresponding author: ganj110@uk.ac.ir
}

associated with the two refill grooves was neglected. Experimental studies of pressure distribution in finite slider bearing with single continuous surface profile on the pads were conducted by Sharma and Pandey [7]. The main goal was to compare the experimental results of pressure distribution in slider bearing with various single continuous surface profiles of pads (cycliodal, cateniodal, polynomial and plane). It was found that the pressure generated by cycliodal profile pad is larger in comparison to the pressure obtained with other profile pads for identical operating conditions. Tello [8] has theoretically studied the regularity of the solution to the Reynolds equation in Rayleigh step type bearings for both compressible and incompressible fluids by employing a rigorous mathematical approach. There are many research works in which the well known Reynolds equation was solved by different numerical schemes in predicting the lubricant pressure field in step bearings [9, 10]. Farmer and Shepherd [11] have analyzed the isothermal operation of the Rayleigh step bearing, considering slip flow condition. The influence of step geometry and degree of slip on the pressure field and bearing performance has been discussed. Naduvinamani and Siddangouda [12] have also studied the effect of surface roughness on the hydrodynamic lubrication of porous step-slider bearings with couple stress fluids theoretically. Rahmani et al. [13] comprehensively studied the Rayleigh step slider bearing including the effect of pressure variations at the boundaries 


\section{Nomenclature}

\begin{tabular}{|llll|}
\hline$b$ & Bearing length & $u^{*} v^{*}$ & Dimensionless velocity components \\
$b_{1}$ & Upstream bearing length & $W$ & Load capacity of bearing \\
$b_{2}$ & Downstream bearing length & $x y$ & Horizontal and vertical coordinates \\
$C_{p}$ & Heat capacity & $x^{*} y^{*}$ & Dimensionless coordinates \\
$F$ & Friction force of bearing & & \\
$h_{1}$ & Upstream film thickness & & Greek symbols \\
& & & Friction coefficient \\
$h_{2}$ & Downstream film thickness & $\eta$ & Modified friction coefficient \\
$h_{3}$ & Step height & $\eta_{M}$ & Dynamic viscosity \\
$K_{f}$ & Thermal conductivity & $\mu$ & Dimensionless dynamic viscosity \\
$L$ & Width of bearing & $\mu^{*}$ & Dynamic viscosity at $T_{1}=40^{\circ} \mathrm{C}$ \\
$o$ & Origin of coordinate & $\mu_{1}$ & Dynamic viscosity at $T_{2}=100{ }^{\circ} \mathrm{C}$ \\
$p$ & Pressure & $\mu_{2}$ & Kinematic viscosity \\
$p^{*}$ & Dimensionless pressure & $\nu$ & Density \\
$P r$ & Prandtl number & $\rho$ & Shear stress \\
$P e$ & Peclet number & $\tau$ & \\
$R e$ & Reynolds number & & Inlet \\
$T$ & Temperature & Subscripts & Maximum \\
$T^{*}$ & Dimensionless temperature & in & Surface \\
$U$ & Runner velocity & max & s \\
$u v$ & Velocity components & & \\
\hline
\end{tabular}

on the bearing hydrodynamic characteristics. The bearing is also optimized considering the lubricant flow rate, friction force and friction coefficient. Lee and Kim [14] calculated the air film temperature of Rayleigh step air foil thrust bearing by solving the Reynolds equation and 3D energy equation with THD boundary conditions at the top foil, thrust disc, and cooling air plenum. By using non-Newtonian fluid as lubricant, the pressure distribution and load capacity for Rayleigh step bearing has also been investigated.

In all of the above studies, the Reynolds equation was solved as the governing equation for calculation of lubricant pressure distribution in bearing lubricant flow. This equation is a simplified form of the momentum equation by neglection of fluid inertia terms. It is clear that under the condition of low lubricant viscosity and high runner surface velocity, this equation may lead to unreliable results. The present study which is a continuation of the work of the second author performed in reference [15], deals with lubricant inertia effect on THD characteristics of Rayleigh step bearings. To this end, the momentum equations coupled with the energy equation are solved with and without inertia terms. Comparison between these results can show the accuracy of Reynolds equation in prediction of step bearing characteristics.

\section{Problem description}

The schematic and coordinate system of Rayleigh slider bearing is shown in Figure 1. The bottom wall of the step bearing moves with constant velocity $U$ (runner velocity). The sudden change in film thickness generates a hydrodynamic pressure field that supports an applied

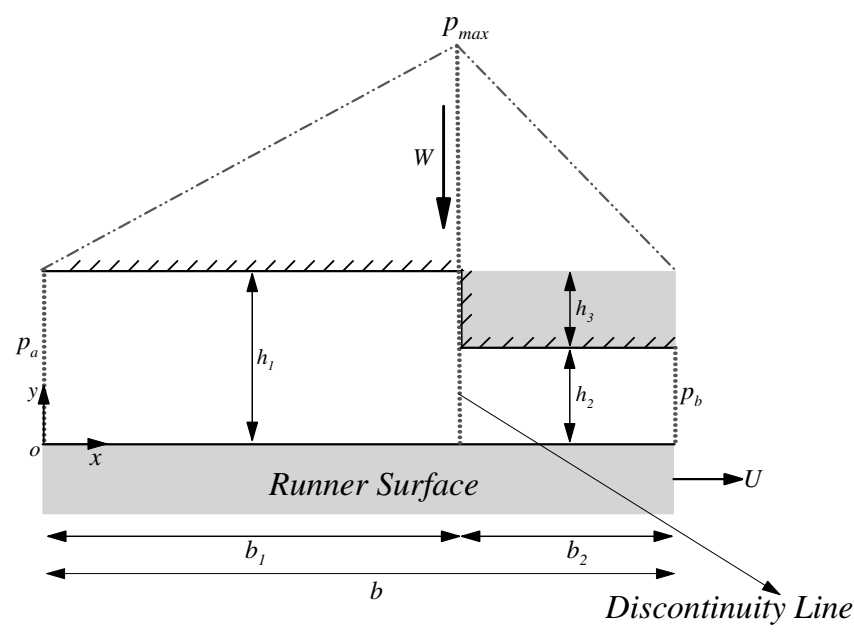

Fig. 1. Sketch of problem geometry.

load $W$. At the inlet section, the oil film enters at $40{ }^{\circ} \mathrm{C}$ with combination of Poiseuille and Couette flows.

Here, $p_{a}$ and $p_{b}$ are oil pressure at the inlet and outlet sections of the bearing, respectively, which are equal to atmospheric pressure. Two important geometrical factors in step bearings are

$$
\begin{gathered}
\varepsilon=\frac{b_{1}}{b} \\
\xi=\frac{h_{1}}{h_{2}}
\end{gathered}
$$

In these explanations, $\varepsilon$ and $\xi$ represent step length and step height ratios, respectively, which are considered to be $\varepsilon=0.718$ and $\xi=1.866$ in the present computations $[16,17]$. 


\section{Theory}

\subsection{Governing equations}

For lubricant flow in bearings, the governing equations which are written for a two-dimensional, steady, incompressible, laminar and variable viscosity flow consist of the continuity, Navier-Stokes and energy equations. These equations can be written as:

$$
\begin{gathered}
\frac{\partial u}{\partial x}+\frac{\partial v}{\partial y}=0 \\
\rho \frac{\partial}{\partial x}\left(u^{2}-\mu \frac{\partial u}{\partial x}\right)+\rho \frac{\partial}{\partial y}\left(u v-\mu \frac{\partial u}{\partial y}\right)=-\frac{\partial p}{\partial x}+F_{B_{x}}(4) \\
\rho \frac{\partial}{\partial x}\left(u v-\mu \frac{\partial v}{\partial x}\right)+\rho \frac{\partial}{\partial y}\left(v^{2}-\mu \frac{\partial v}{\partial y}\right)=-\frac{\partial p}{\partial y}+F_{B_{y}} \\
\frac{\partial}{\partial x}\left(u T-\frac{k_{f}}{\rho C_{p}} \frac{\partial T}{\partial x}\right)+\frac{\partial}{\partial y}\left(v T-\frac{k_{f}}{\rho C_{p}} \frac{\partial T}{\partial y}\right)=\frac{\mu}{\rho C_{p}} \phi
\end{gathered}
$$

where $F_{B_{x}}$ and $F_{B_{y}}$ in momentum equations represent the viscous source terms as follows [16]:

$$
\begin{aligned}
& F_{B_{x}}=\frac{\partial}{\partial x}\left(\mu \frac{\partial u}{\partial x}\right)+\frac{\partial}{\partial y}\left(\mu \frac{\partial v}{\partial x}\right) \\
& F_{B_{y}}=\frac{\partial}{\partial x}\left(\mu \frac{\partial u}{\partial y}\right)+\frac{\partial}{\partial y}\left(\mu \frac{\partial v}{\partial y}\right)
\end{aligned}
$$

Also $\phi$ denotes the viscous dissipation term according to following formula:

$$
\phi=\left[2\left(\frac{\partial u}{\partial x}\right)^{2}+2\left(\frac{\partial v}{\partial y}\right)^{2}+\left(\frac{\partial u}{\partial y}+\frac{\partial v}{\partial x}\right)^{2}\right]
$$

The variation of fluid viscosity with temperature is also considered in the present analysis based on the Vogel equation:

$$
\mu=\mu_{i n} e^{-\beta\left(T-T_{i n}\right)}
$$

In this expression, $\beta$ is the temperature-viscosity coefficient of the lubricant. The value of $\beta$ is determined using two given viscosity values at $T_{1}$ and $T_{2}$ as follows [16]:

$$
\beta=\frac{\ln \left(\mu_{1} / \mu_{2}\right)}{T_{2}-T_{1}}
$$

\subsection{Boundary conditions}

The entire domain is fully flooded, such that oil pressure at the inlet and outlet sections of the bearing is set to zero atmospheric gauge pressure. Also the no-slip condition is employed on all boundary solid walls.
At the inlet section, oil enters into the bearing with uniform temperature of $T_{i n}$ and a specified velocity distribution which is a combination of the Poiseuille and Couette flows with a pressure gradient which is obtained iteratively. For this purpose, the Reynolds equation is solved numerically for computing a good initial value for lubricant pressure gradient. About the inlet oil temperature, it should be mentioned that for a given feeding oil temperature, the lubricant temperature at the inlet section will be increasing by decreasing in the value of minimum oil film thickness, which will in turn, affect bearing performance at high thrust loads. But as a simplified assumption, it is assumed that the inlet oil temperature is constant for step bearing running under different thrust loads. It is evident that this assumption leads to accurate results when bearing loads are light. Besides, at the outlet section, zero axial gradients for all dependent variables are employed.

Also, the temperature of runner surface is assumed to be constant, which is computed from a zero global heat flux balance at the fluid and runner surface interface [10]:

$$
\int_{0}^{b}\left(\frac{\partial T}{\partial y}\right)_{y=0} \mathrm{~d} x=0
$$

Finally, the adiabatic condition is imposed on the bearing solid surface.

\subsection{Nondimensional forms of the governing equations}

In numerical solution of the set of governing equations including the continuity, momentum and energy, the following dimensionless parameters are used to obtain the nondimensional forms of these equations [15]:

$$
\begin{aligned}
\left(x^{*}, y^{*}\right) & =\left(\frac{x}{h_{1}}, \frac{y}{h_{1}}\right),\left(u^{*}, v^{*}\right)=\left(\frac{u}{U}, \frac{v}{U}\right), \\
p^{*} & =\frac{p}{\rho U^{2}}, \mu^{*}=\frac{\mu}{\mu_{i n}}, T^{*}=\frac{\rho C_{p} h_{1}}{\mu_{i n} U} \frac{h_{1}}{b}\left(T-T_{i n}\right) \\
R e & =\frac{\rho U h_{1}}{\mu_{i n}}, P e=\frac{\rho C_{p} U h_{1}}{k_{f}}=\frac{U h_{1}}{\alpha}
\end{aligned}
$$

In these definitions, $\alpha$ is the thermal diffusivity of the lubricant and $\mu_{i n}$ is the inlet lubricant viscosity.

The nondimensional forms of the governing equations in the Cartesian coordinate system are as follows:

$$
\frac{\partial u^{*}}{\partial x^{*}}+\frac{\partial v^{*}}{\partial y^{*}}=0
$$

$$
\begin{array}{r}
\frac{\partial}{\partial x^{*}}\left(u^{* 2}-\frac{\mu^{*}}{R e} \frac{\partial u^{*}}{\partial x^{*}}\right)+\frac{\partial}{\partial y^{*}}\left(u^{*} v^{*}-\frac{\mu^{*}}{R e} \frac{\partial u^{*}}{\partial y^{*}}\right) \\
=-\frac{\partial p^{*}}{\partial x^{*}}+F_{B_{x}}^{*}
\end{array}
$$




$$
\begin{array}{r}
\frac{\partial}{\partial x^{*}}\left(u^{*} v^{*}-\frac{\mu^{*}}{R e} \frac{\partial v^{*}}{\partial x^{*}}\right)+\frac{\partial}{\partial y^{*}}\left(v^{* 2}-\frac{\mu^{*}}{R e} \frac{\partial v^{*}}{\partial y^{*}}\right) \\
=-\frac{\partial p^{*}}{\partial y^{*}}+F_{B_{y}}^{*} \\
\frac{\partial}{\partial x^{*}}\left(u^{*} T^{*}-\frac{1}{P e} \frac{\partial T^{*}}{\partial x^{*}}\right)+\frac{\partial}{\partial y^{*}}\left(v^{*} T^{*}-\frac{1}{P e} \frac{\partial T^{*}}{\partial y^{*}}\right) \\
=\mu^{*}\left(\frac{h_{2}}{b}\right) \phi^{*}
\end{array}
$$

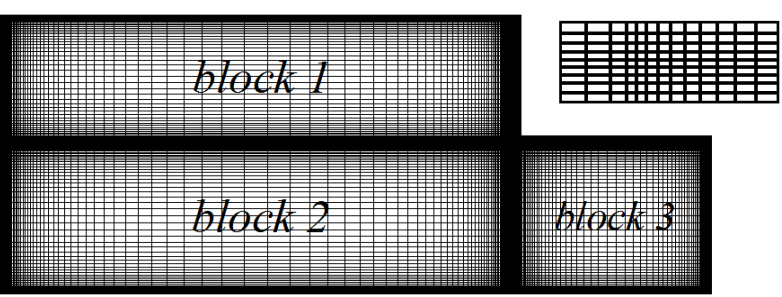

Fig. 2. A schematic of grid generation.

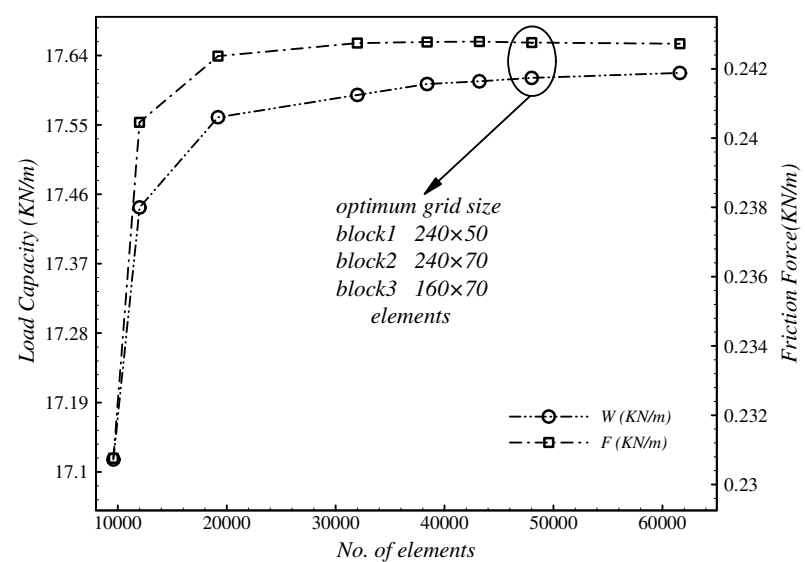

Fig. 3. Solution dependence on grid size, $\varepsilon=0.718, \xi=1.866$, $b=0.08 \mathrm{~m}, R e=413$.

\section{Solution procedure}

tion can be calculated as follows:

$$
\mu^{*}=\mathrm{e}^{-\beta \frac{\mu_{i} U}{\rho C_{p} h_{1}}\left(\frac{b}{h_{1}}\right) T *}
$$

\subsection{Main physical quantities}

The main physical quantities of interest in lubrication study are the load capacity and friction force.

The load capacity of the step bearing per unit width is obtained by further integration of lubricant pressure distribution:

$$
\frac{W}{L}=\int_{0}^{b} p \mathrm{~d} x
$$

The friction force of the step bearing is calculated by the shear stress on the bottom wall as follows:

$$
\frac{F}{L}=\int_{0}^{b} \tau_{s} \mathrm{~d} x
$$

where:

$$
\tau_{s}=\mu \frac{\partial u(x, y)}{\partial x} \quad \text { at } \quad y=0
$$

Now, the friction coefficient can be calculated from the following relation:

$$
\eta=\frac{F}{W}
$$

Finite difference forms of the continuity, momentum and energy equations were obtained by integrating over an elemental cell volume with staggered control volumes for the $x$ - and $y$-velocity components. Other variables of interest were computed at the grid nodes. The nondimensionalized governing equations were discretized by using the hybrid scheme and numerically solved by the SIMPLE algorithm of Patankar and Spalding [18]. Numerical solutions were obtained iteratively by the line-by-line method progressing in axial direction. The iterations were terminated when the sum of the absolute residuals was less than $10^{-4}$ for each equation. Numerical calculations were performed by writing a computer program in FORTRAN.

As shown in Figure 2, the computational domain is divided into three blocks, each having $N_{x}$ points in $x$-direction and $N_{y}$ points in $y$-direction. The mesh is nonuniform in $x$ - and $y$-directions with the largest cell (being the one furthest away from the step). The grid refinement around the step is necessary to capture the occurrence of the recirculation and other flow changes due to the sudden change in geometry. As the result of grid tests for obtaining the grid-independent solutions, an optimum grid for each block is shown in Figure 3 and discretization details for all of the cases presented in Table 1.

\section{Validation of computational results}

To test the validity of the present numerical results, computations were carried out for two test cases and the 
Table 1. Grid independence study, $\varepsilon=0.718, \xi=1.866$, $b=0.08 \mathrm{~m}, R e=413$.

\begin{tabular}{ccc}
\hline Grid size & Load capacity Friction force \\
$($ block1). (block2). (block3) & $\left(\right.$ KN.m $\left.{ }^{-1}\right)$ & $\left(\right.$ KN.m $\left.{ }^{-1}\right)$ \\
\hline$(100 \times 20)(100 \times 40) .(60 \times 40)$ & 17.116 & 0.2307 \\
$(120 \times 20)(120 \times 40) .(80 \times 40)$ & 17.443 & 0.2404 \\
$(140 \times 30)(140 \times 50) .(100 \times 50)$ & 17.560 & 0.2423 \\
$(200 \times 40)(200 \times 60) .(120 \times 60)$ & 17.588 & 0.2426 \\
$(200 \times 50)(200 \times 70) .(120 \times 70)$ & 17.602 & 0.2427 \\
$(220 \times 50)(220 \times 70) .(140 \times 70)$ & 17.607 & 0.2427 \\
$(240 \times 50)(240 \times 70) .(160 \times 70)$ & 17.610 & 0.2427 \\
$(240 \times 60)(240 \times 80) .(180 \times 80)$ & 17.612 & 0.2427 \\
\hline
\end{tabular}

computed results were compared with theoretical results of other investigators. The lubricant pressure and temperature distributions along the axial direction are shown in Figures 4 and 5 . It should be mentioned that the values of bearing geometrical parameters and also the lubricant properties are given in the related literatures $[9,10]$.

Figure 4 shows the lubricant pressure distribution of the bearing studied by Dobrica and Fillon [10]. The generated hydrodynamic pressure by the sudden contraction in flow domain is clearly seen in this figure, such that the lubricant maximum pressure takes place just at the entrance of narrow gap of the bearing. However, the agreement between the present numerical results and numerical data is quite good.

To validate the present computations for lubricant temperature, the isotherm lines for a step bearing analyzed in reference [10] are plotted in Figure 5. The lubricant temperature rise along the flow direction which is due to viscous dissipation is clearly seen in this figure. Besides this figure shows that the adiabatic boundary condition for the bearing surface is almost realistic such that a good consistency is seen between the isotherm patterns plotted in Figure 5b based on the present numerical results with those obtain in reference [10] shown in Figure 5a where the conjugate problem containing conduction-convection heat transfer in solid element and oil flow was solved. However, comparison between these figures indicates an overestimation about $8{ }^{\circ} \mathrm{C}$ in computation of maximum oil temperature which is due to changing a conjugate problem to a simple one with simplifying assumptions. If one focuses to Figure 5, it is seen that the maximum difference between the present numerical results with those obtained in reference [10] takes place near to the pad surface where the effect of combined conduction-convection heat transfer in the conjugate problem is enhanced.

In another test case, the computed lubricant pressure distribution and also the temperature distribution on the bearing surface are compared with the numerical results of Hideki in Figure 6. A similar trend which was observed before for the lubricant pressure in previous case test is also seen in Figure 6, with a very good consistency between the present numerical results with those found by Hideki [9].

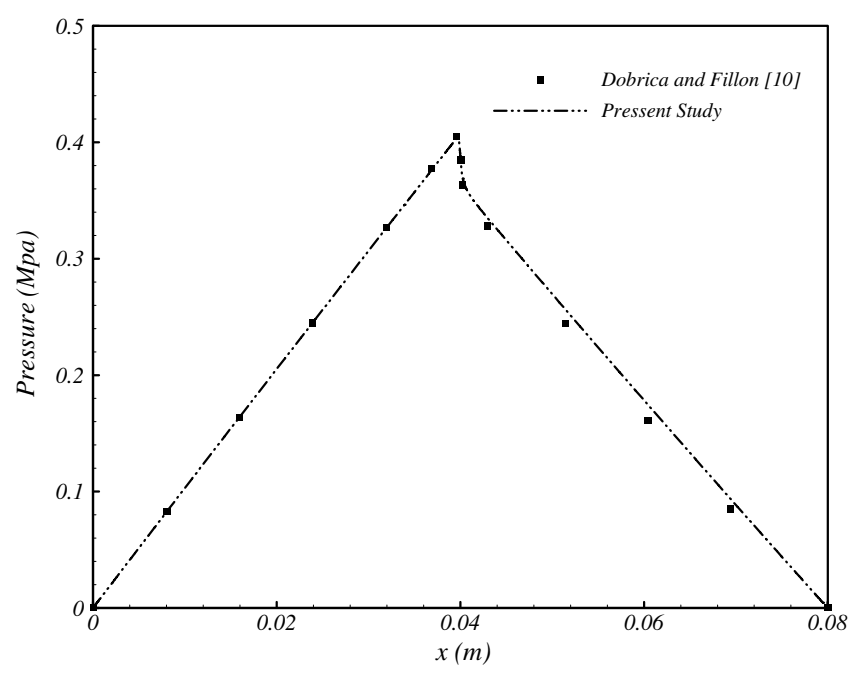

Fig. 4. Lubricant pressure distribution on the bottom wall of the bearing studied by Dobrica and Fillon [10], $\varepsilon=0.5, \xi=2$, $b=0.08 \mathrm{~m}, h_{2}=240 \mu \mathrm{m}, U=30 \mathrm{~m} . \mathrm{s}^{-1}$.

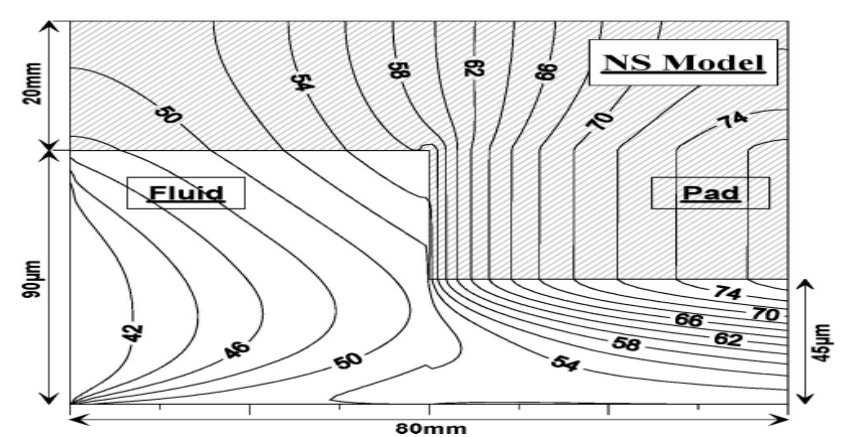

(a)

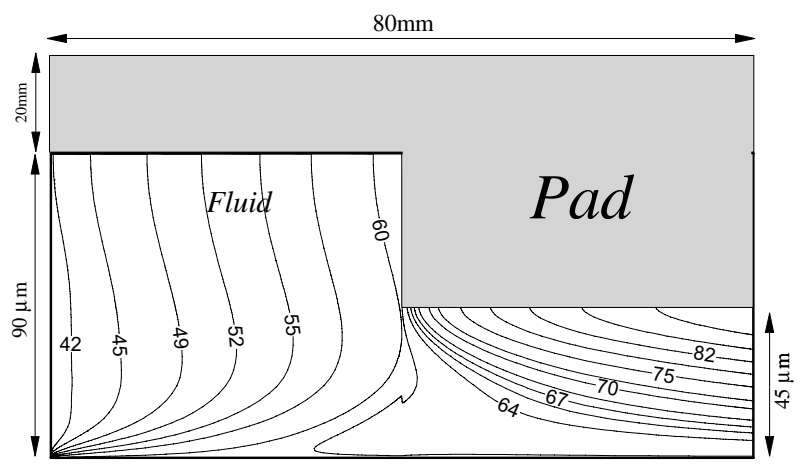

(b)

Fig. 5. Temperature distribution in the bearing studied by Dobrica and Fillon [10], $\varepsilon=0.5, \xi=2, b=0.08 \mathrm{~m}, h_{2}=$ $45 \mu \mathrm{m}, U=30 \mathrm{~m} \cdot \mathrm{s}^{-1}$. (a) Reference [10]. (b) Present work.

Figure 6 depicts that the lubricant temperature increases along the flow direction because of the viscous dissipation in both domains upstream and downstream sides of the step. But Figure 6 shows a very high rate of temperature increase after the step location in comparison to the upstream region which is as a result of high dissipation rate in lubricant film with small thickness and 


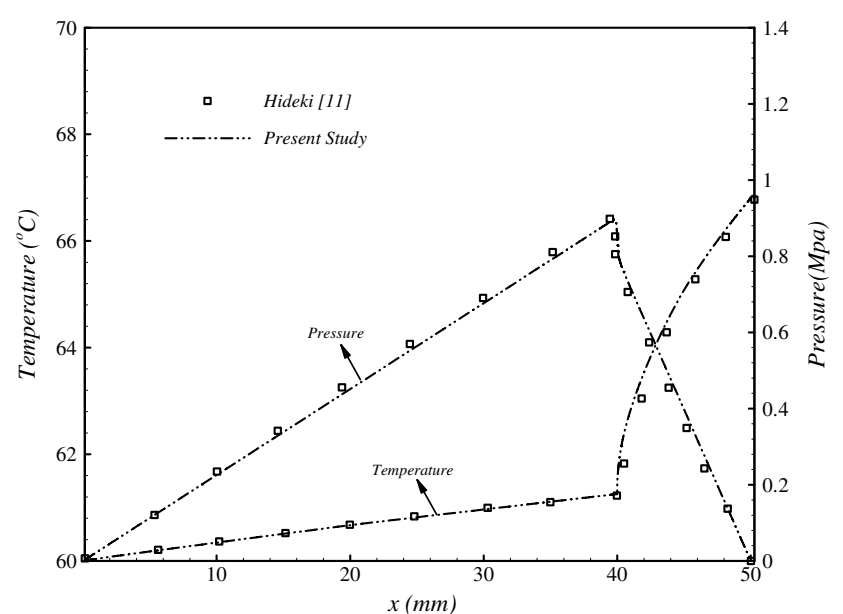

Fig. 6. Lubricant pressure distribution on the bottom wall and temperature distribution on the top wall of the bearing studied by Hideki [9], $\varepsilon=0.8, \xi=2, b=50 \mathrm{~mm}, h_{2}=100 \mu \mathrm{m}$, $U=30 \mathrm{~m} \cdot \mathrm{s}^{-1}$.

Table 2. Bearing parameters and lubricant properties.

\begin{tabular}{cccc}
\hline Parameters & Units & Present work & Reference [10] \\
\hline$b$ & $\mathrm{~m}$ & 0.08 & 0.08 \\
$h_{1}$ & $\mu \mathrm{m}$ & $93-480$ & $30-960$ \\
$h_{2}$ & $\mu \mathrm{m}$ & $50-257$ & $15-480$ \\
$T_{\text {in }}$ & ${ }^{\circ} \mathrm{C}$ & 40 & 40 \\
$\rho$ & $\mathrm{kg} \cdot \mathrm{m}^{-3}$ & 860 & 860 \\
$C_{p}$ & ${\mathrm{~J} . \mathrm{kg}^{-1} \cdot{ }^{\circ} \mathrm{C}}$ & 2000 & 2000 \\
$K_{f}$ & $\mathrm{~W}\left(\mathrm{~m}^{-1} \cdot{ }^{\circ} \mathrm{C}\right)$ & 0.13 & 0.13 \\
$\mu_{\text {at }} 40{ }^{\circ} \mathrm{C}$ & $\left(\mathrm{N} . \mathrm{s}^{-2}\right)$ & 0.03 & 0.03 \\
$\mu_{\text {at } 100}{ }^{\circ} \mathrm{C}$ & $\left(\mathrm{N} . \mathrm{s} \cdot \mathrm{m}^{-2}\right)$ & 0.0045 & 0.0045 \\
$\varepsilon$ & - & 0.718 & 0.5 \\
$\xi$ & - & 1.866 & 2 \\
$R e$ & - & $70-413$ & - \\
\hline
\end{tabular}

consequently with high velocity gradient. However, a good agreement is observed between the present numerical results and the numerical findings by Hideki [9].

\section{Results and discussion}

In this paper, THD characteristics of Rayleigh step bearings are obtained by numerical solution of the NavierStocks and energy equations using the CFD technique. An attempt is made for comparing the lubricant pressure and temperature distributions and also some other dependent variables with and without considering the fluid inertia. All of the subsequent figures are about a Rayleigh step bearing whose properties and geometrical parameters are given in Table 2.

First the oil flow pattern inside the bearing is shown in Figure 7 by plotting the fluid velocity vectors. The adverse pressure gradient in the upstream flow domain before the step location which leads to hydrodynamic pressure generation causes a concave shape for velocity distribution. Such that the velocity distribution changes

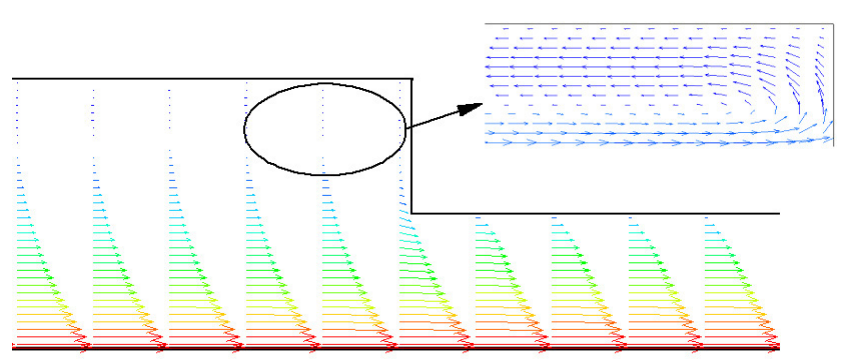

Fig. 7. Velocity vectors in step bearing lubricant flow, $h_{2}=$ $275 \mu \mathrm{m}, R e=413$.

to convex shape after the step where there is a favourable pressure gradient. Behind the step surface near to the stationary wall, a circulated flow domain appears which is due to the effects of both viscous friction and positive pressure gradient in this region. As another result that can be seen from Figure 7, one can notice to the almost stationary flow region in block 1 (see Fig. 2). Therefore, the lubricant average velocity across blocks 2 and 3 remains approximately constant.

The lubricant pressure distributions on the bottom bearing wall along the axial direction are plotted in Figure 8 for two cases of considering and neglecting the fluid inertia effect at different values of the Reynolds number and the minimum oil film thickness.

All curves plotted in Figure 8 depict a same pattern for pressure distribution such that there is an increasing trend in lubricant pressure in upstream region to the bearing step after which the lubricant pressure decreases up to the bearing outlet section. This figure shows two opposite effects for inertia terms on lubricant pressure, such that neglection of fluid inertia underestimates the value of lubricant pressure in upstream region of the bearing step and then it switches to overestimation downstream the step.

Comparison between the curves plotted in Figure 8 shows that the inertia term has more effect on hydrodynamic pressure at high Reynolds number, especially for high values of lubricant minimum film thickness. Such that the maximum different between the pressure distribution for these two cases is seen in $R e=413$ for $h_{2}=275 \mu \mathrm{m}$ with an error of about $8 \%$ in predicting lubricant maximum pressure. Besides, comparison between the curves plotted in Figure 8 reveals that the amount of hydrodynamic pressure generation in step bearing increases in a high rate by decreasing in the minimum film thickness.

Temperature distributions on the top wall of the bearing surface and also at the oil film section $y=h / 4$ along the axial direction for two different runner velocities and minimum oil film thicknesses are plotted in Figures 9 and 10 with inertia effect and in inertialess case.

These figures present that the lubricant inertia has more effect on lubricant pressure in comparison to temperature. It is seen that neglection of fluid inertia introduces a very small underestimation in temperature 


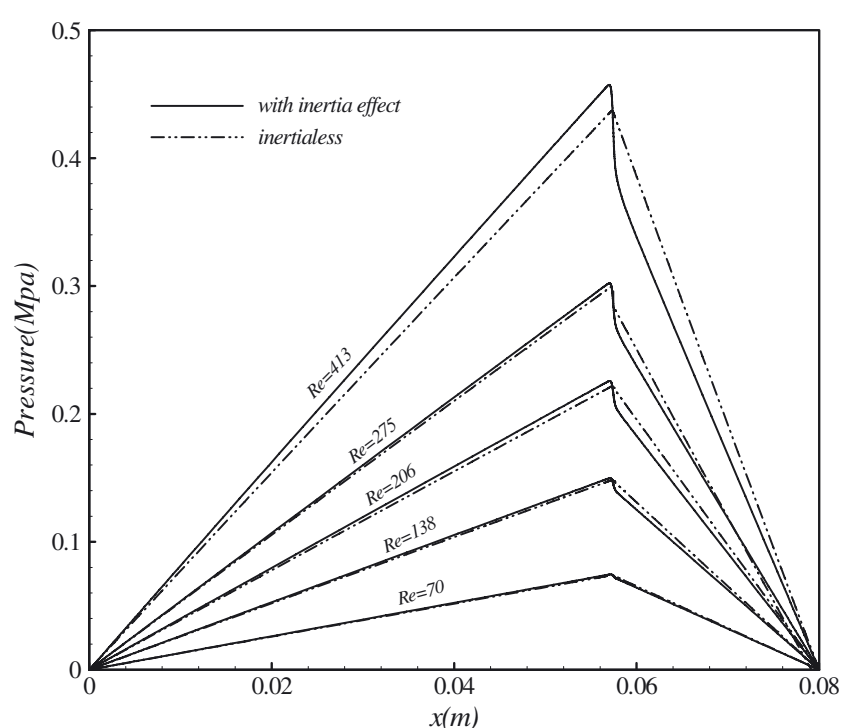

(a)

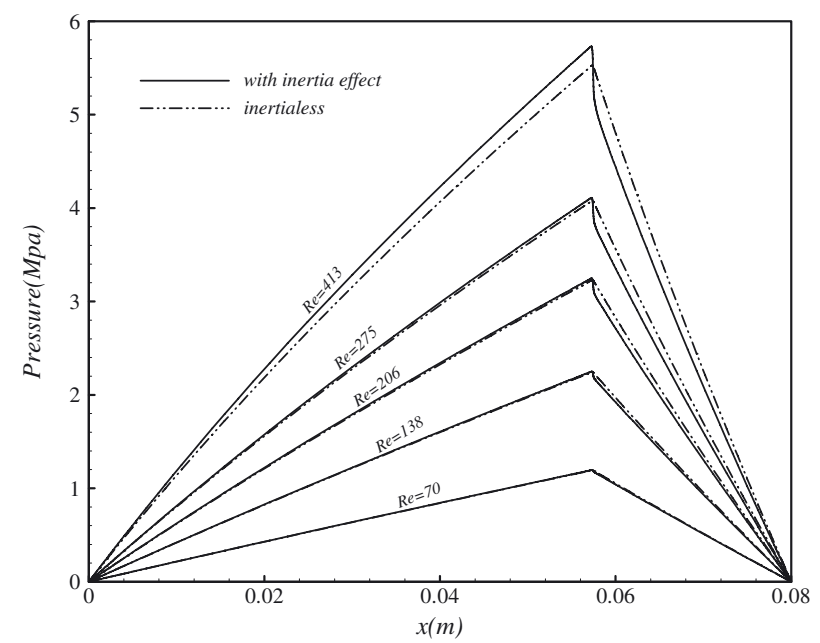

(b)

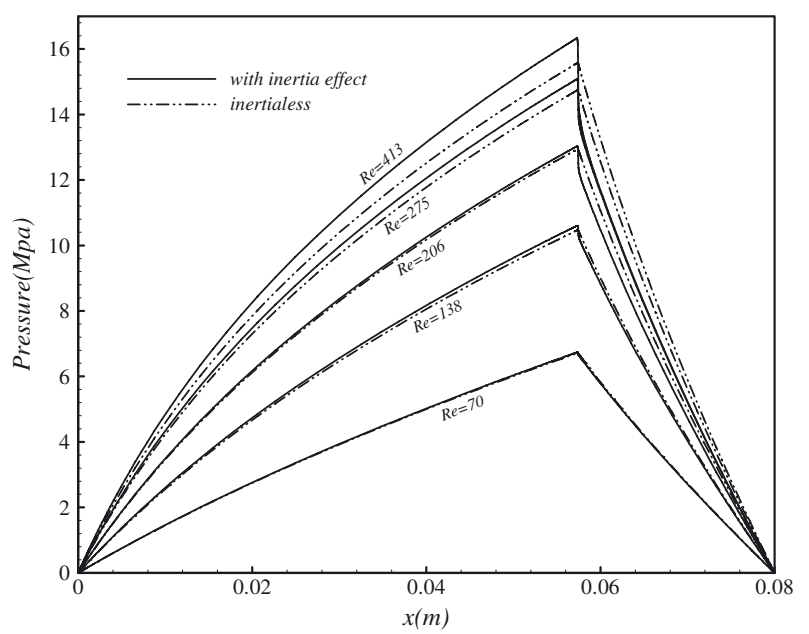

(c)

Fig. 8. Lubricant pressure distribution on the bottom wall of the bearing at three different values for the lubricant minimum film thickness. (a) $h_{2}=275 \mu \mathrm{m}$. (b) $h_{2}=100 \mu \mathrm{m}$. (c) $h_{2}=$ $50 \mu \mathrm{m}$.

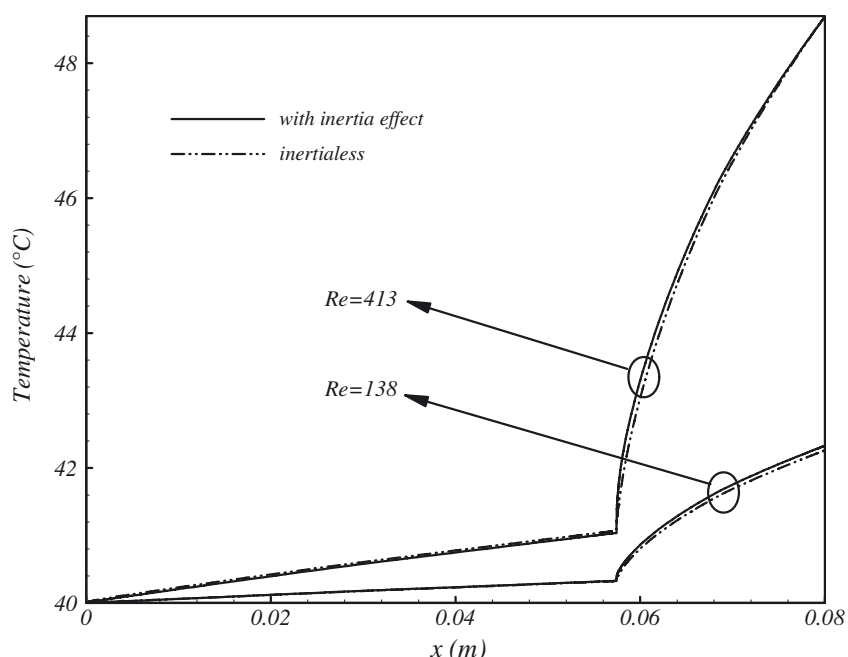

(a)

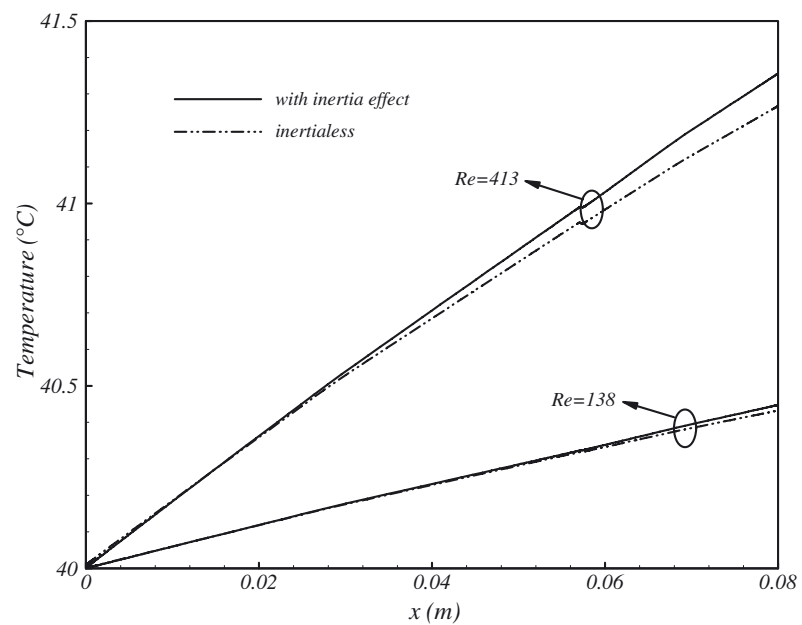

(b)

Fig. 9. Lubricant temperature distributions, $h_{2}=275 \mu \mathrm{m}$. (a) Temperature distribution on the top wall of the bearing. (b) Temperature distribution at the axial section $y=h / 4$.

calculation, especially at small values of the Reynolds number and minimum oil film thickness.

The effect of inertia in maximum lubricant pressure is studied in Figure 11 with plotting the variation of $P_{\max }$ with Reynolds number in inertialess case and in the case of considering inertia effect.

It is seen that $P_{\max }$ increases with increasing in Reynolds number with this fact that neglection of inertia effect underestimates the value of maximum pressure with about $14 \%$ maximum error that occurs at $R e=413$.

Similar curves but for maximum lubricant temperature are plotted in Figure 12. This figure also shows that inertia has not considerable effect in temperature calculation. However, it can be concluded that the fluid inertia causes an increase in computing the value of $T_{\max }$, such that Figure 12 shows about $0.4 \%$ for maximum error that takes place at $R e=300$ and not at $R e_{\max }$. It can be due to nonlinear relation between the dissipation term in energy equation with the lubricant velocity field. 


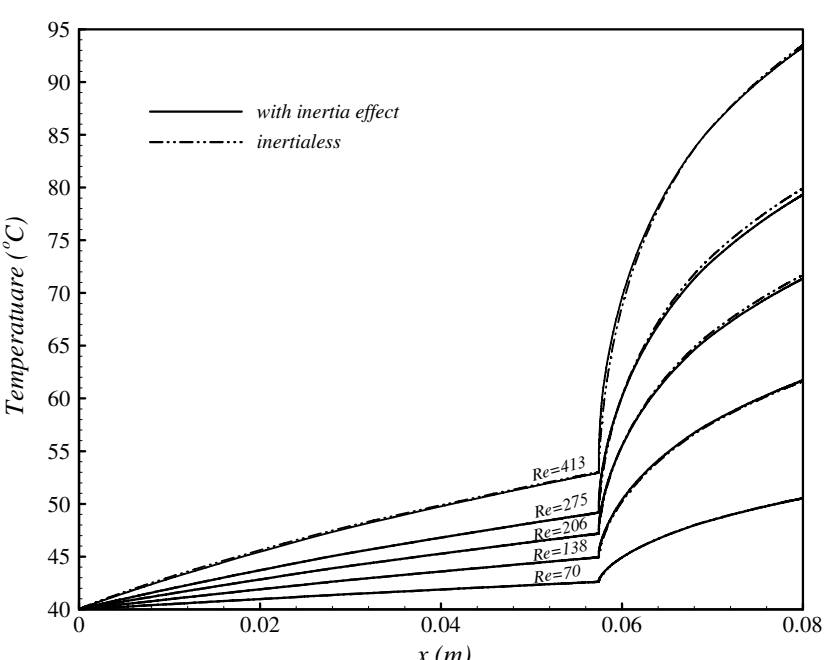

(a)

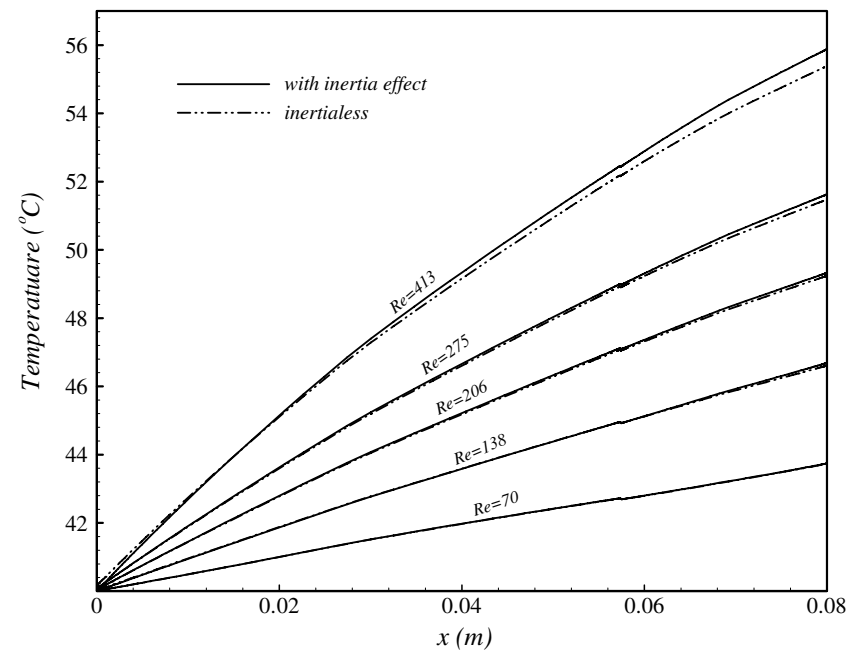

(b)

Fig. 10. Lubricant temperature distributions, $h_{2}=100 \mu \mathrm{m}$. (a) Temperature distribution on the top wall of the bearing.

(b) Temperature distribution at the axial section $y=h / 4$.

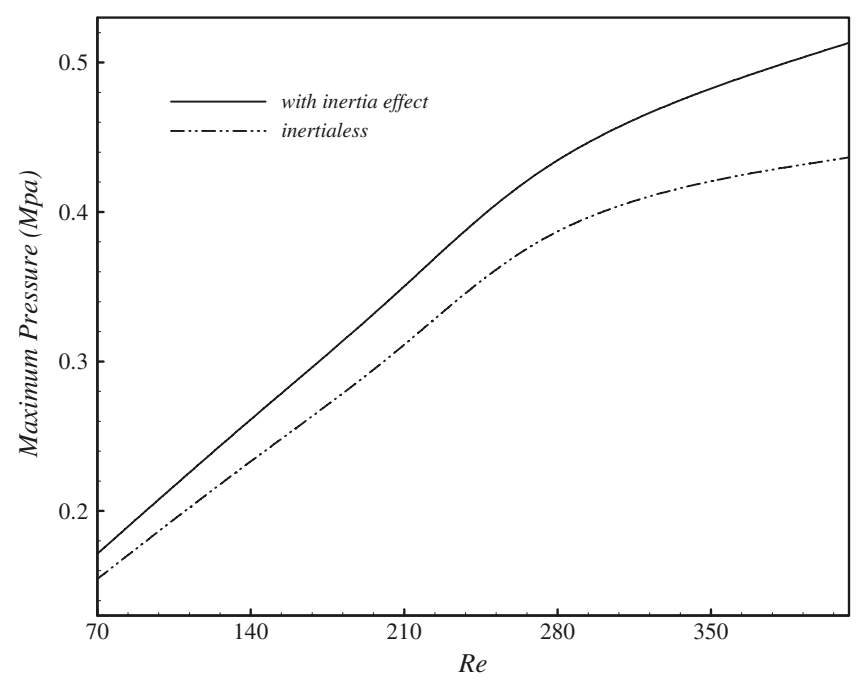

Fig. 11. Variation of lubricant maximum pressure with Reynolds number, $h_{2}=275 \mu \mathrm{m}$.

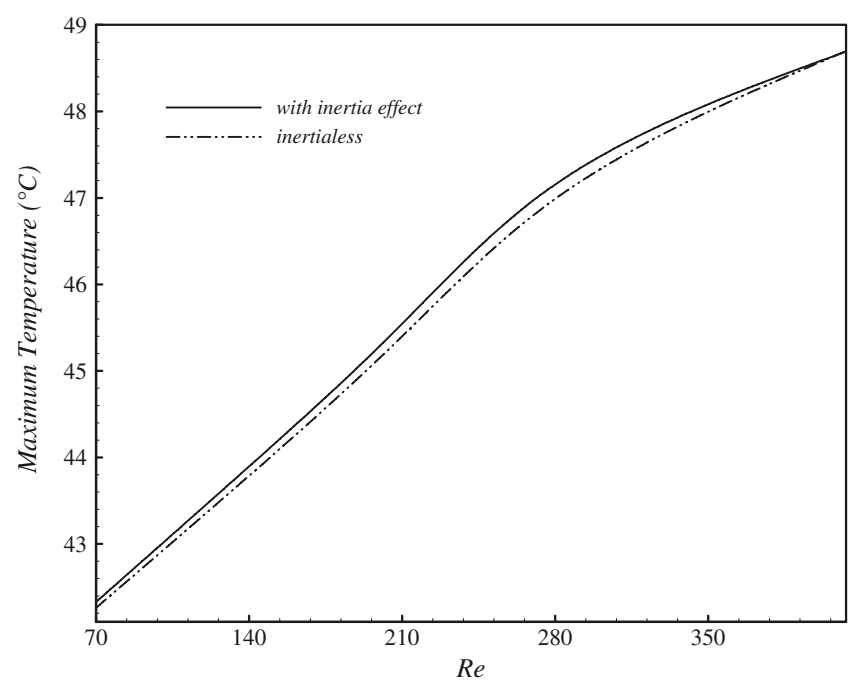

Fig. 12. Variation of lubricant maximum temperature with Reynolds number, $h_{2}=275 \mu \mathrm{m}$.

Finally the effects of fluid inertia on bearing load capacity and also on bearing friction force are shown in Figure 13. This figure shows small inertia effect on bearing load capacity with maximum error of $2.5 \%$, but a great inertia effect on bearing friction force with error $_{\max } \sim 8 \%$. Besides, it is seen that inertialess assumption underestimates the values of both bearing load and friction force such that the amount of underestimation increases by increasing in $R e$.

Computations of load capacity, friction force and friction coefficient are repeated for smaller values of the lubricant minimum film thickness i.e., $h_{2}=100 \mu \mathrm{m}$ and $h_{2}=50 \mu \mathrm{m}$, and the variations of these parameters with Re are plotted in Figures 14 and 15. Similar trends are found for load capacity and friction force such that the rate of increase in these parameters with $R e$ in much more in the case with small value for minimum film thickness. But it is seen that the friction coefficient decreases with $R e$. It is due to this fact that the rate of increase in load capacity with $R e$ is greater than that is for friction force, and according to the definition of the friction coefficient (Eq. (23)), this parameter has decreasing trend with $R e$, such that neglection of inertia enhances this behavior, especially at high values for minimum film thickness. If one compares the curves plotted in Figures 13c, 14c and 15c, it can be seen that for all values of the oil minimum film thickness, the friction coefficient has a decreasing trend with Reynolds number such that, the rate of decreasing is much more for step bearings running under low Reynolds number. Also it is seen that for step bearings running under small minimum film thickness, the rate of decrease in friction coefficient with increasing $R e$ is much more.

\section{Conclusion}

A CFD based study is conducted to investigate the lubricant inertia effect on THD characteristics of the 


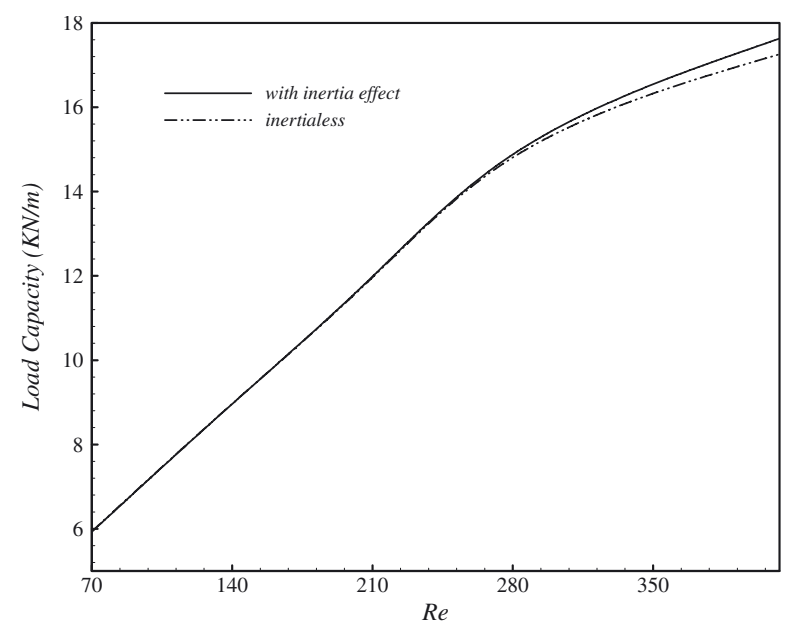

(a)

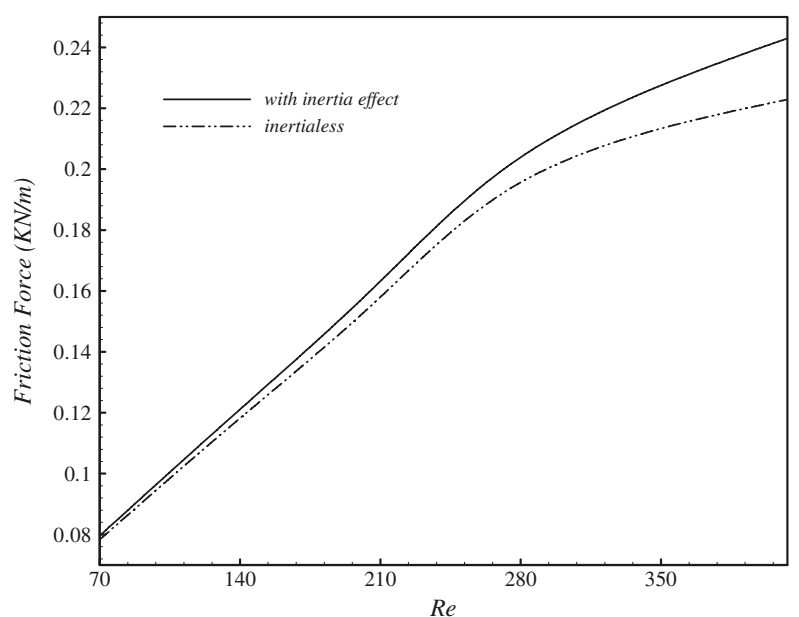

(b)

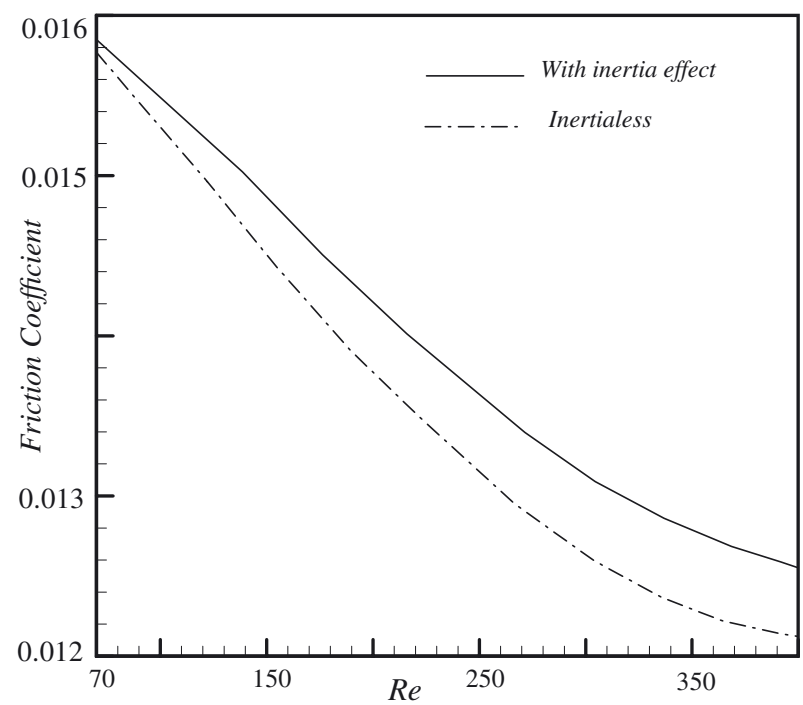

(c)

Fig. 13. Variations of the load capacity, friction force and friction coefficient with Reynolds number, $h_{2}=275 \mu \mathrm{m}$. (a) Load capacity. (b) Friction force. (c) Friction coefficient.

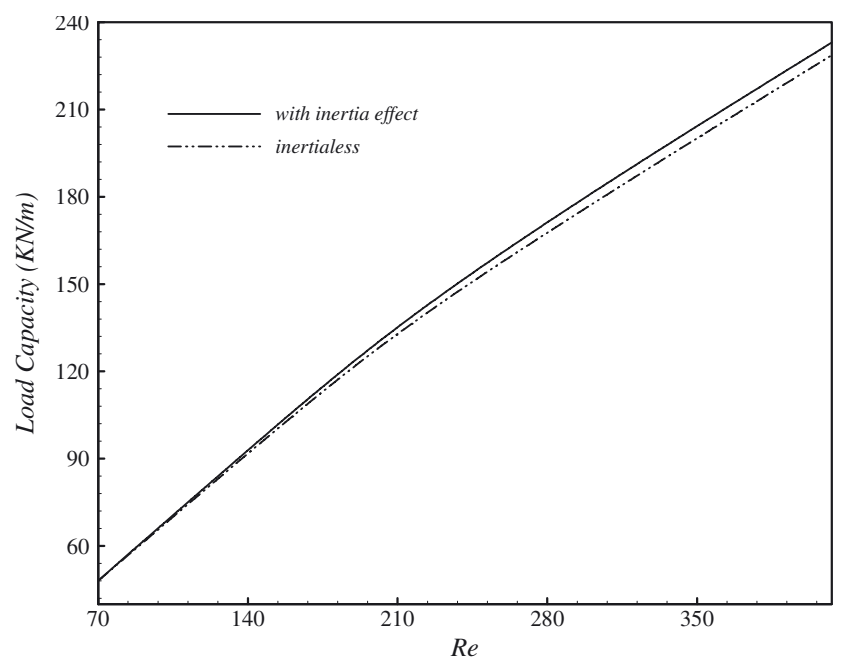

(a)

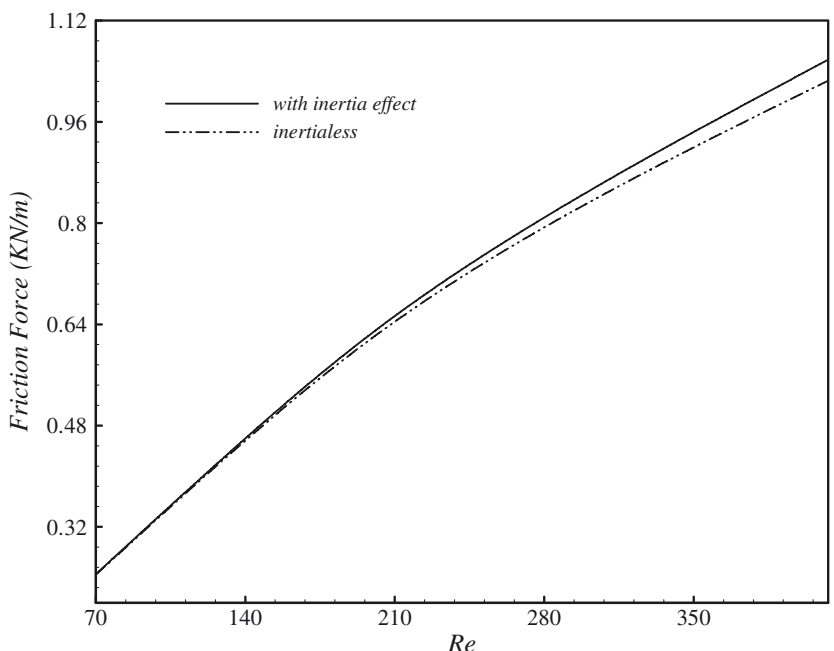

(b)

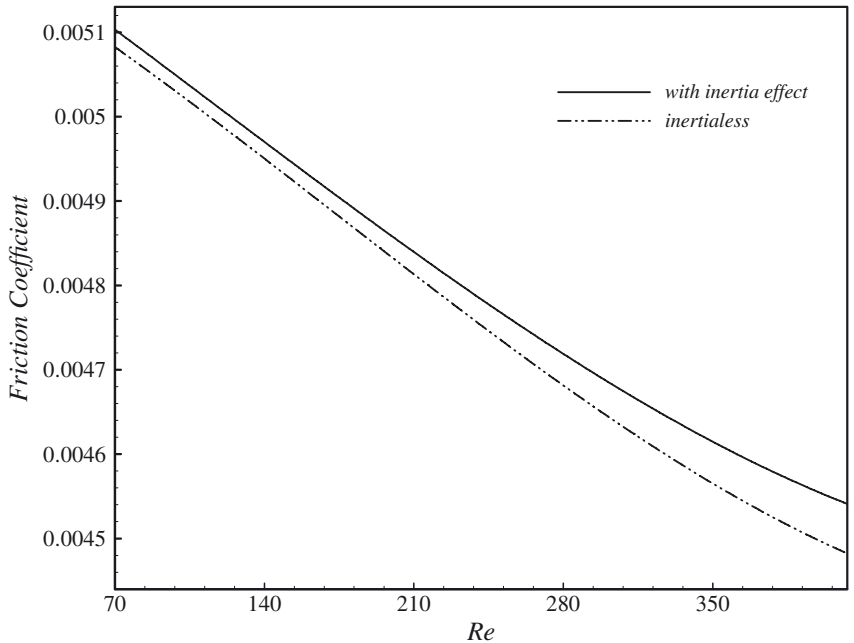

(c)

Fig. 14. Variations of the load capacity, friction force and friction coefficient with Reynolds number, $h_{2}=100 \mu \mathrm{m}$. (a) Load capacity. (b) Friction force. (c) Friction coefficient. 


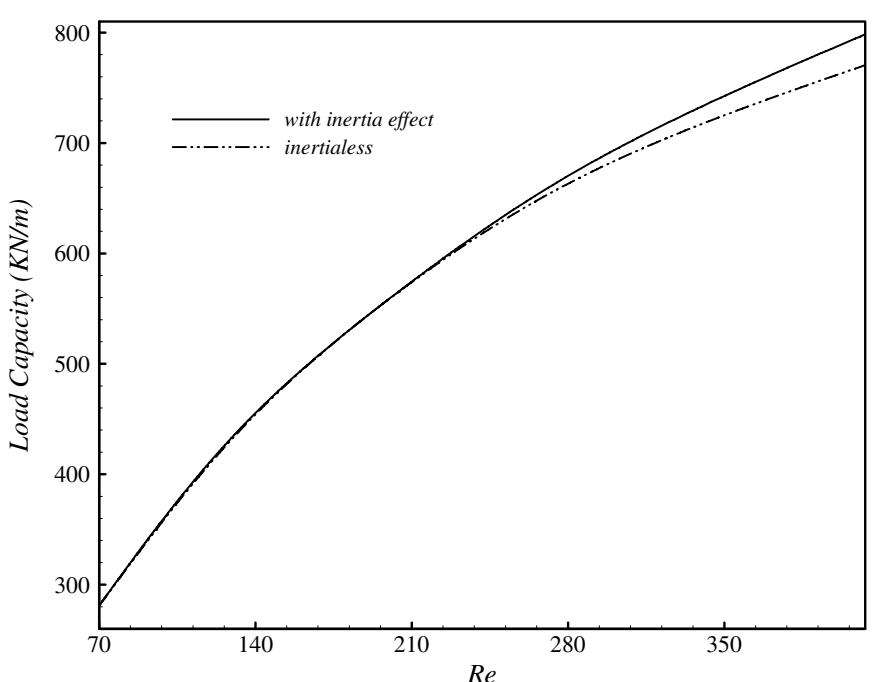

(a)

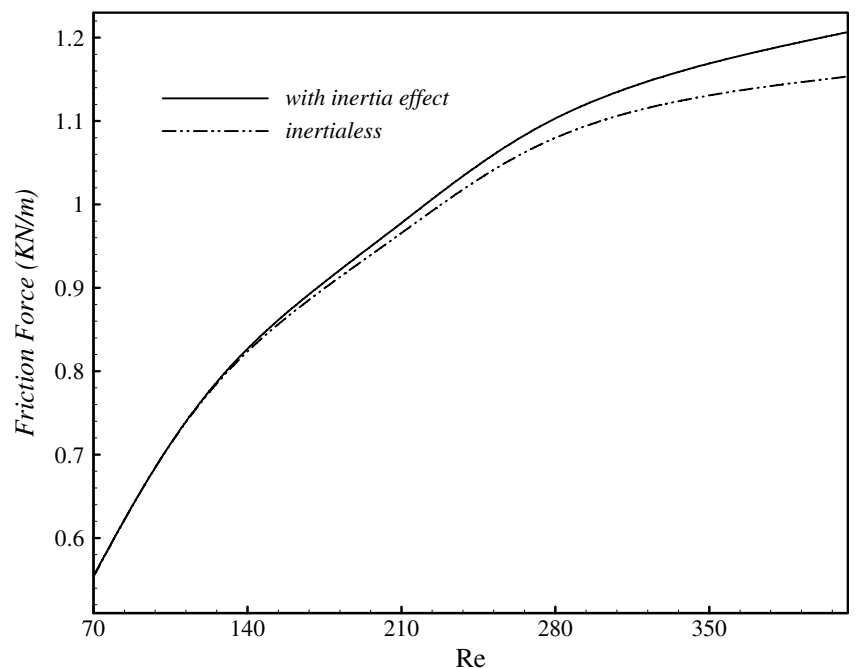

(b)

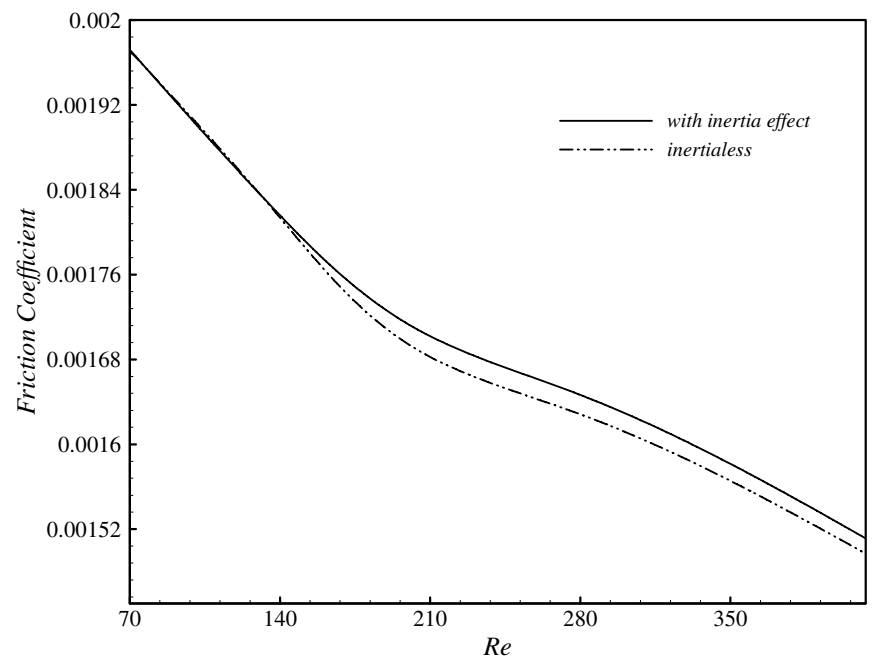

(c)

Fig. 15. Variations of the load capacity, friction force and friction coefficient with Reynolds number, $h_{2}=50 \mu \mathrm{m}$. (a) Load capacity. (b) Friction force. (c) Friction coefficient.
Rayleigh step bearings. To this end, the set of governing equations is solved with and without considering the inertia terms in momentum equations. Discretized forms of the governing equations are obtained by the control volume method and solved by the SIMPLE algorithm. Numerical results show that THD characteristics of step bearings are affected by fluid inertia, and that lubricant pressure field is more affected than the lubricant temperature. Numerical results reveal that the values of lubricant maximum pressure and lubricant maximum temperature and also the values of bearing load capacity and friction force increase by the lubricant inertia effect. Such that solution of the set of governing equations without inertia term lead to underestimation in the values of the above mentioned parameters, especially when step bearings are running under high runner surface velocity and large minimum film thickness.

\section{References}

[1] D. Dowson, A generalized Reynolds equation for fluid film lubrication, Int. J. Mech. Sci. 4 (1962) 159-170

[2] H.A. Ezzat, S.M. Rohde, A study of thermohydrodynamic performance of finite slider bearings, ASME J. Lubric. Technol. 95 (1973) 298-307

[3] R. Boncompain, M. Fillon, J. Frene, Analysis of thermal effects in hydrodynamic bearings, ASME J. Tribol. 108 (1986) 219-224

[4] J.Y. Auloge, P. Bourgin, B. Gay, The optimum design of one-dimensional bearings with non Newtonian lubricants, Trans. ASME J. Lubric. Technol. 105 (1983) 391-39

[5] M. Fillon, M.M. Khonsari, Thermohydrodynamic design charts for tilting-pad journal bearings, ASME J. Tribol. 118 (1996) 232-238

[6] M. Arghir, A. Alsayed, D. Nicolas, The finite volume solution of the Reynolds equation of lubrication with film discontinuities, Int. J. Mech. Sci. 44 (2002) 2119-2132

[7] R.K. Sharma, R.K. Pandey, Experimental studies of pressure distribution in finite slider bearing with single continuous surface profiles on the pad, Tribol. Int. (2009) 42 1040-1045

[8] J.I. Tello, Regularity of solutions to a lubrication problem with discontinuous separation data, Nonlinear Anal. 53 (2003) 1167-77

[9] O. Hideki, Thermohydrodynamic lubrication analysis method of step bearings, IHI Eng. Rev. 38 (2005) 6-10

[10] M. Dobrica, M. Fillon, Reynolds' model suitability in simulating Rayleigh step bearing thermohydrodynamic problems, Tribol. Trans. 48 (2005) 522-530

[11] D.G. Farmer, J.J. Shepherd, Slip flow in the gaslubricated Rayleigh step-slider bearing, Int. J. App. Mech. Eng. 11 (2006) 593-608

[12] N.B. Naduvinamani, A. Siddangouda, Effect of surface roughness on the hydrodynamic lubrication of porous step-slider bearings with couple stress fluids, Tribol. Int. 40 (2007) 780-793 
[13] R. Rahmani, A. Shirvani, H. Shirvani, Analytical analysis and optimisation of the Rayleigh step slider bearing, Tribol. Int. 42 (2009) 666-674

[14] D. Lee, D. Kim, Three-dimensional thermohydrodynamic analyses of Rayleigh step air foil thrust bearing with radially arranged bump foils, Tribology. Trans. 54 (2011) 432-448

[15] S.A. Gandjalikhan Nassab, Inertia effect on thermohydrodynamic characteristics of journal bearings, Proc. ImechE 219 (2005), Part J, J. Tribol. 459-467
[16] M. Khonsari, E.R. Booser, Applied Tribology 2e: Bearing design and lubrication, John Willy \& Sons. Ltd., 2008, pp. $29-41$

[17] W. Jianming, J. Gaobing, The optimum design of the Rayleigh slider bearing with a power law fluid, Wear 129 (1989) 1-11

[18] S.V. Patankar, D.B. Spalding, A calculation procedure for heat, mass and momentum transfer in threedimensional parabolic flows, Int. J. Heat Mass Transf. 15 (1972) 1787-1806 